ISSN 1392-3196 / e-ISSN 2335-8947

Zemdirbyste-Agriculture, vol. 102, No. 4 (2015), p. 417-422

DOI 10.13080/z-a.2015.102.053

\title{
Effect of tree pruning intensity on the yield and fruit quality of the sour cherry
}

\author{
Krzysztof RUTKOWSKI, Zofia ZYDLIK, Eugeniusz PACHOLAK \\ University of Life Sciences \\ Dąbrowskiego 159, 60-594 Poznań, Poland \\ E-mail: krzysztof.rutkowski@au.poznan.pl
}

\begin{abstract}
The aim of the study was to estimate the effect of' tree pruning intensity on the yielding and quality of fruits of the sour cherry (Prunus cerasus L.) cultivar 'Łutówka'. An experiment with a different intensity of tree pruning was carried out in the years 2006-2010. The study material consisted of one-year old budded plants of the first class: cv. 'Łutówka' type IR2, on Prunus mahaleb rootstock, planted in the spring of 2001 and 2002 spaced $4.0 \times 1.3 \mathrm{~m}$ giving a total of 1920 trees $\mathrm{ha}^{-1}$. In the plantation, three pruning regimes were applied: 1) no pruning (only sanitation felling was applied), 2) moderate traditional pruning (consisting of the shortening of branches), 3) intensive pruning (by removing branches older than three years). Tree yielding significantly depended on the weather conditions of the experimental years. With increasing pruning intensity the yield decreased. Tree pruning and its intensity influenced fruit quality measured by the weight of 100 fruits, fruit firmness, acidity and total soluble solids. Colour variation of 'Łutówka' skin was assigned the following parameters $\mathrm{L}^{*}, \mathrm{a}^{*}, \mathrm{~b}^{*}, \mathrm{C}^{*}$ and $\mathrm{h}^{\circ}$. Their changes varied depending on the year and orchard.
\end{abstract}

Key words: crown form, cultivar, fruit quality, pruning, Prunus cerasus, yield.

\section{Introduction}

Poland is one of the largest producers of sour cherries in the world (Szpadzik et al., 2009; Sredojević et al., 2011). The annual harvest is about 180 thousand tones, except in the years when spring frosts damage the flowers or fruitlets (Mika et al., 2011). Intensification of fruit production is frequently measured by the tree plantation density. In sour cherry orchards, the recommended number of trees per hectare ranges from 667 to 2760 . Such dense plantation requires an adequate rootstock and tree canopy, which may significantly influence the yield and quality of fruits.

For intensive sour cherry orchards, it is frequently recommended to apply a narrow spindle like or axial tree canopy (Gonda, 2006; Hrotkó et al., 2008 b; Hrotkó, 2009; Magyar, Hrotkó, 2013), while for orchards with a smaller number of trees, a circular, regulated tree is recommended (Gonda, Such, 2008; Hrotkó, 2013). The formation of an adequate tree canopy requires different pruning intensities which exert an influence on a number of tree physiological processes connected with the growth, yield and quality of fruits (Buler, Mika, 2009; Jankiewicz, Lipecki, 2011).

In the narrow spindle and in the axial crown forms, being the most common ones, intensive pruning is applied by removing branches older than three years and leaving a tenon from which young shoots grow out and after 2-3 years they are removed again leaving place for new shoots (Hrotkó et al., 2008 a). In orchards with a circular, almost natural crown, a cleaning pruning is applied to remove excess of branches or to shorten the branches. This procedure forces the shoots to shoot out closer to the centre of the crown (Mika et al., 2011).

Sour cherry tree of the cultivar 'Łutówka' requires a particular pruning method because it does not produce very many flower buds on the shoots while the leaf buds are in the peak parts of trees. The pruning repeated in the successive years leads to shoot denudation of leaves and, in consequence, to a decrease in the size and quality of yields (Mika et al., 2011).

The objective of the study was to estimate the effect of pruning intensity of ' $€ u$ utówka' sour cherry trees on spring frost damage, yield and quality of fruit.

\section{Material and methods}

Our experiment was carried out in 2006-2010 in the Department of Fruit-Growing of Poznań University of Life Sciences at the Agricultural and Fruit Growing Experimental Farm in Przybroda, Poland.

The experiment consisted of two stages. Oneyear old budded plants were planted in the spring 2001 and 2002 spaced $4.0 \times 1.3 \mathrm{~m}$, which gave a total of 1920 trees per ha. The investigated material consisted of oneyear old budded plants of the sour cherry (Prunus cerasus L.) cultivar 'Łutówka', type IR2 on Prunus mahaleb. All trees had been produced in one nursery and they met the first class quality. In autumn, before the orchard establishment, farmyard manure (40 tons), potassium (200 kg ha-1 $\mathrm{K}_{2} \mathrm{O}$ ) and phosphorus (185 $\mathrm{kg} \mathrm{ha}^{-1} \mathrm{P}_{2} \mathrm{O}_{5}$ ) fertilization were applied. The plantation was established 
as a randomized split block design with four replications including a total of forty trees.

In the orchard, three intensities of' tree pruning were used: 1) no pruning (only sanitary felling was applied), 2) moderate traditional pruning (consisting of the shortening of branches by $1 / 3$ of length) and 3) intensive pruning (by removing branches older than 3 years).

Every year, before vegetation, nitrogen fertilization was applied in the form of ammonium nitrate $\left(50 \mathrm{~kg} \mathrm{ha}^{-1} \mathrm{~N}\right)$. Tree pruning was carried out every year after fruit harvest in the second half of August. In the orchard, between rows, turf was cultivated, while in the tree rows herbicide fallow was maintained. Diseases and pests were controlled according to the protection program for commercial orchards.

The yield was estimated from each tree separately and its quality was expressed by the firmness of fruit flesh, total soluble solids and titratable acidity (results were expressed in percentage of malic acid) in the mass of 100 fruits. The sample size was 200 fruits picked from ten trees per replication at the height ranging from 130 to $160 \mathrm{~cm}$, from the external part of the tree. Fruit firmness was determined for 25 sour cherries (whole fruits with the peel) using a penetrometer fruit pressure tester model FT 02 (Facchini Srl, Italy), with a 2.5-mm diameter tip and the results were expressed in grams $(\mathrm{g})$. The total soluble solid content of the juice was determined with a digital refractometer PR-101 $\alpha$ ("Atago", Japan) on 25 cherries selected randomly from each replicate and expressed in \%. The colour was measured with a handheld tristimulus reflectance colorimeter Minolta CR-100 (Minolta Corp., USA) and recorded using a CIE L* $\mathrm{a}^{*}$ and $b^{*}$ uniform colour space. Numerical values of $a^{*}$ and $\mathrm{b}^{*}$ were converted into Hue angle $\left(\mathrm{h}^{\circ}=\tan ^{-1} \mathrm{~b}^{*} / \mathrm{a}^{*}\right)$ and chroma $\left(\right.$ Chroma $\left.=\left(a^{*} 2+b^{*} 2\right)^{1 / 2}\right)($ Eysiak, 2012; Łysiak et al., 2014).

At the time of blooming in 2006, the weather was favourable and sunny. In the year 2007, spring frosts occurred twice and caused damage to flowers and fruitlets (Table 1).

Table 1. Influence of spring frost on flower damage depending on tree pruning intensity in 2007

\begin{tabular}{|c|c|c|c|c|}
\hline $\begin{array}{l}\text { Year of } \\
\text { planting }\end{array}$ & Treatment & $\begin{array}{c}\% \text { damaged flowers } \\
(27042007)\end{array}$ & $\begin{array}{c}\% \text { set fruits } \\
(08052007)\end{array}$ & $\begin{array}{c}\text { Yield } \\
\text { in } 2007 \\
\end{array}$ \\
\hline \multirow{3}{*}{2001} & Without pruning & 9.22 & 66.57 & $7.16 \mathrm{a}^{*}$ \\
\hline & Moderate traditional pruning & 7.62 & 60.59 & $6.85 \mathrm{a}$ \\
\hline & Intensive pruning & 13.47 & 54.98 & $5.88 \mathrm{a}$ \\
\hline \multirow{3}{*}{2002} & Without pruning & 6.98 & 56.55 & $6.73 \mathrm{a}$ \\
\hline & Moderate traditional pruning & 10.68 & 52.54 & $8.35 \mathrm{a}$ \\
\hline & Intensive pruning & 6.08 & 56.29 & $6.74 \mathrm{a}$ \\
\hline 2001 & & 10.10 & 60.71 & $6.63 \mathrm{a}$ \\
\hline 2002 & & 7.91 & 55.13 & $7.27 \mathrm{a}$ \\
\hline
\end{tabular}

Note. ${ }^{*}$ - different letters in the same column and for the same year represent significant differences at $5 \%$ level of significance.

The greatest temperature drop occurred during the nights from the $20^{\text {th }}$ to the $22^{\text {nd }}$ of April (Fig. 1). The drop of temperature lasted almost 4 hours and caused significant damage to set flowers.

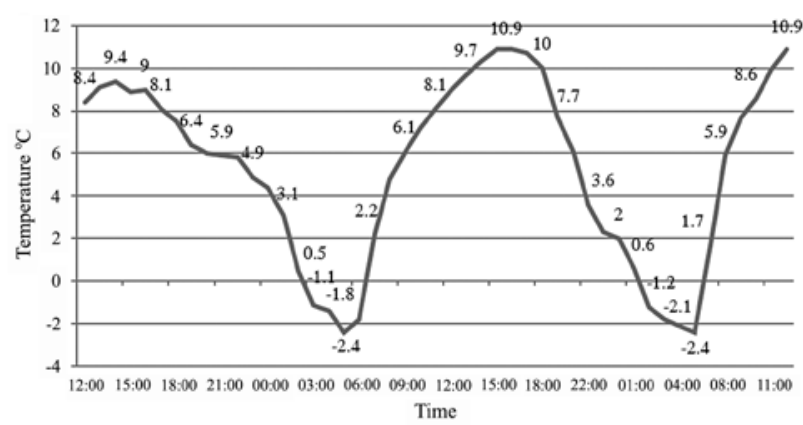

Figure 1. Course of air temperature measured at $2 \mathrm{~m}$ height at night from $20^{\text {th }}$ to $22^{\text {nd }}$ April, 2007

Spring frost occurred on the night from the $30^{\text {th }}$ April to the $1^{\text {st }}$ May, the temperature was $-1.6^{\circ} \mathrm{C}$ for 5 hours (Fig. 2).

The weather conditions during bloom time in 2008 were unfavourable for pollination and fertilization. The average temperature during the day was below $10^{\circ} \mathrm{C}$ and it was raining. In the year 2009, flowering conditions were optimal but hailstorms decreased the yield and quality of fruits. The low productivity in the year 2010 was caused by unfavourable climatic conditions during flowering. Similar weather conditions during bloom time were in Przybroda in 2004-2005, which significantly decreased the sour cherry yield.

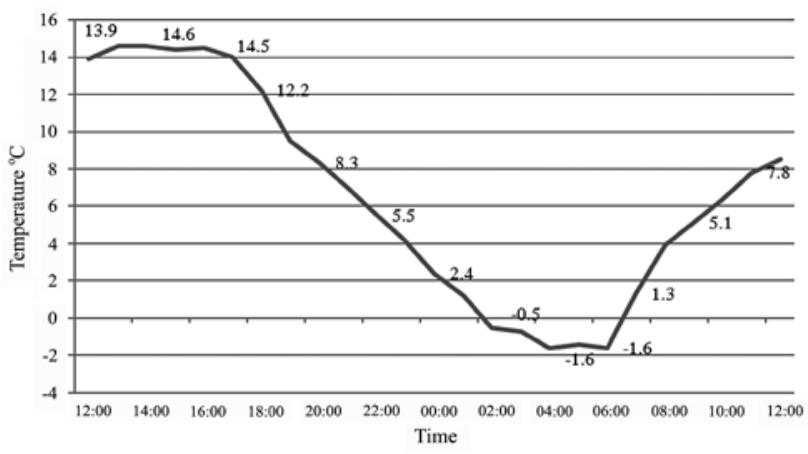

Figure 2. Course of air temperature measured at $2 \mathrm{~m}$ height on the night from $30^{\text {th }}$ April to $1^{\text {st }}$ May, 2007

Results were statistically processed by multifactorial analysis of variance using programs Statistica 12 and STAT. Significance of differences between the treatments was estimated based on Duncan's test at a significance level of $\alpha=0.05$.

\section{Results and discussion}

Tree yielding significantly depended on the weather conditions during the bloom time in the experimental years 2006-2010. In the same experiment, the mean bloom damage in the year 2005 ranged between $39.5 \%$ in the orchard established in 2001 and $41.8 \%$ in the orchard established in 2002. Spring frost contributed to a decrease in the sour cherry yield (Zydlik et al., 2006). According to Davarynejad et al. (2014), a satisfactory yield can be obtained when $25-30 \%$ of the flowers set fruit. 
Sour cherries are frequently damaged by spring frost which reduces the yield (Szpadzik et al., 2008; Stepulaitiené et al., 2013). Unfavourable weather conditions decreased sour cherry yield. It was most visible in the orchard established in 2001 where the mean yield in the vegetation periods 2007 and 2010 was significantly lower than in 2006, 2008 and 2009 (Table 2). A similar tendency occurred in an orchard established in 2002 (Table 3). Application of pruning regimes in the orchard established in 2001 (Table 1) and in 2002 (Table 2) did exert significant effect on the total yield. Increased pruning intensity decreased the yield. Trees which had not been pruned were characterized by a significantly higher yield in comparison to the pruned ones. This fact is consistent with the statement of Jankiewicz and
Lipecki (2011) who reported that tree pruning decreased tree yield. As a result of pruning, the number of shoots and buds decreases together with some nutrients accumulated in the removed parts. Thus, competition for nutrients occurs, which results in shoots growing with a lower number of buds. The influence of pruning on fruit yield determined in this trial supports the results of many earlier experiments carried out by Mika et al. (2003) obtained from apple and by Ikinci (2014) obtained from peach showing that pruning of trees reduced the fruiting volume. Previous studies have already shown that sour cherry yields were higher for the rectangular shaped trees than in the spindle canopy (Flore, Layne, 1990). Spindle trees are characterised by poorer yields in the later years due to the lack of permanent basal branches

Table 2. Effect of tree pruning intensity on the yield and fruit quality of sour cherry tree cultivar ' $L u$ tówka' planted in 2001

\begin{tabular}{|c|c|c|c|c|c|c|}
\hline \multirow{3}{*}{ Treatment } & \multicolumn{5}{|c|}{ Years } & \multirow{2}{*}{$\begin{array}{l}\text { Mean for years } \\
2006-2010\end{array}$} \\
\hline & 2006 & 2007 & 2008 & 2009 & 2010 & \\
\hline & \multicolumn{6}{|c|}{ Yield kg tree ${ }^{-1}$} \\
\hline Without pruning & $8.01 \mathrm{a}^{*}$ & $7.16 \mathrm{a}$ & $8.62 \mathrm{a}$ & $9.85 \mathrm{~b}$ & $6.08 \mathrm{~b}$ & $7.95 \mathrm{~b}$ \\
\hline Moderate traditional pruning & $8.24 \mathrm{a}$ & $6.85 \mathrm{a}$ & $8.73 \mathrm{a}$ & $6.60 \mathrm{a}$ & $4.00 \mathrm{a}$ & $6.88 \mathrm{a}$ \\
\hline Intensive pruning & $9.19 \mathrm{a}$ & $5.88 \mathrm{a}$ & $6.37 \mathrm{a}$ & $7.09 \mathrm{a}$ & $3.55 \mathrm{a}$ & $6.42 \mathrm{a}$ \\
\hline \multirow[t]{2}{*}{ Mean for year } & $8.48 \mathrm{c}$ & $6.63 \mathrm{a}$ & $7.91 \mathrm{c}$ & $7.85 \mathrm{c}$ & $4.54 \mathrm{a}$ & \\
\hline & \multicolumn{6}{|c|}{100 fruit weight $g$} \\
\hline Without pruning & $534.2 \mathrm{a}$ & $449.2 \mathrm{ab}$ & $483.2 \mathrm{a}-\mathrm{c}$ & $508.5 \mathrm{~b}-\mathrm{d}$ & $611.2 \mathrm{a}$ & $517.3 \mathrm{a}$ \\
\hline Moderate traditional pruning & $527.2 \mathrm{a}$ & $442.0 \mathrm{a}$ & $516.5 \mathrm{~b}-\mathrm{e}$ & $553.5 \mathrm{de}$ & $637.0 \mathrm{a}$ & $535.2 \mathrm{ab}$ \\
\hline Intensive pruning & $498.3 \mathrm{a}$ & $472.2 \mathrm{~b}$ & 568.5 ef & $524.8 \mathrm{~b}-\mathrm{e}$ & $660.4 \mathrm{a}$ & $544.8 \mathrm{~b}$ \\
\hline \multirow[t]{2}{*}{ Mean for year } & $519.9 \mathrm{~b}$ & $454.5 \mathrm{a}$ & $522.7 \mathrm{~b}$ & $528.9 \mathrm{~b}$ & $636.2 \mathrm{c}$ & \\
\hline & \multicolumn{6}{|c|}{ Firmness g } \\
\hline Without pruning & $248.5 \mathrm{a}$ & $216.2 \mathrm{a}$ & $201.7 \mathrm{a}$ & $187.0 \mathrm{a}$ & $199.5 \mathrm{a}$ & $210.6 \mathrm{a}$ \\
\hline Moderate traditional pruning & $280.5 \mathrm{a}$ & $232.7 \mathrm{a}$ & $209.7 \mathrm{a}$ & $187.0 \mathrm{a}$ & $173.0 \mathrm{a}$ & $216.6 \mathrm{a}$ \\
\hline Intensive pruning & $263.0 \mathrm{a}$ & $207.0 \mathrm{a}$ & $191.2 \mathrm{a}$ & $175.5 \mathrm{a}$ & $170.2 \mathrm{a}$ & $201.4 \mathrm{a}$ \\
\hline \multirow[t]{2}{*}{ Mean for year } & $264.0 \mathrm{c}$ & $218.6 \mathrm{~b}$ & $200.9 \mathrm{ab}$ & $183.1 \mathrm{a}$ & $180.9 \mathrm{a}$ & \\
\hline & \multicolumn{6}{|c|}{ Soluble solid content ${ }^{\circ}$ Brix } \\
\hline Without pruning & $16.07 \mathrm{~b}$ & $14.85 \mathrm{a}$ & $17.32 \mathrm{a}$ & $13.10 \mathrm{a}$ & $15.67 \mathrm{a}$ & $15.40 \mathrm{~b}$ \\
\hline Moderate traditional pruning & $15.85 \mathrm{ab}$ & $14.35 \mathrm{a}$ & $16.65 \mathrm{a}$ & $12.85 \mathrm{a}$ & $14.75 \mathrm{a}$ & $14.89 \mathrm{a}$ \\
\hline Intensive pruning & $15.25 \mathrm{a}$ & $14.35 \mathrm{a}$ & $16.72 \mathrm{a}$ & $13.35 \mathrm{a}$ & $14.37 \mathrm{a}$ & $14.81 \mathrm{a}$ \\
\hline \multirow[t]{2}{*}{ Mean for year } & $15.72 \mathrm{c}$ & $14.51 \mathrm{~b}$ & $16.90 \mathrm{~d}$ & $13.10 \mathrm{a}$ & $14.93 \mathrm{~b}$ & \\
\hline & \multicolumn{6}{|c|}{ Acidity as the malic content $\%$} \\
\hline Without pruning & $1.76 \mathrm{~b}$ & $1.49 \mathrm{~b}$ & $1.89 \mathrm{~b}$ & $2.01 \mathrm{a}$ & $1.67 \mathrm{a}$ & $1.76 \mathrm{~b}$ \\
\hline Moderate traditional pruning & $1.62 \mathrm{a}$ & $1.34 \mathrm{a}$ & $1.72 \mathrm{a}$ & $1.72 \mathrm{a}$ & $1.71 \mathrm{a}$ & $1.62 \mathrm{a}$ \\
\hline Intensive pruning & $1.70 \mathrm{ab}$ & $1.44 \mathrm{ab}$ & $1.72 \mathrm{a}$ & $1.77 \mathrm{a}$ & $1.85 \mathrm{a}$ & $1.70 \mathrm{~b}$ \\
\hline Mean for year & $1.69 \mathrm{~b}$ & $1.42 \mathrm{a}$ & $1.78 \mathrm{bc}$ & $1.83 \mathrm{c}$ & $1.74 \mathrm{bc}$ & \\
\hline
\end{tabular}

Note. ${ }^{*}$ - different letters in the same column and for the same year represent significant differences at $5 \%$ level of significance.

(Hrotkó, 2013). Callesen (1997) received a better yield of control trees than in the hedgerow system with a greater volume of canopy. We conclude that moderate or intensive pruning probably reduced the fruiting volume because cv. 'Łutówka' exhibited poor growth and low ability to propagate shoots. On the other hand, no significant differences were found between moderate and an intensive pruning (Tables 2-3).

Tree pruning and its intensity did not exert any major effect on fruit quality measured by the firmness and extract content. Significant effect of the applied tree pruning was found in relation to the mass of 100 fruits (Table 3). Independently from the orchard age, the fruits reached a mass exceeding $500 \mathrm{~g}$ per 100 fruits, which is consistent with the statement of Szpadzik et al. (2010) who reported that cv. 'Łutówka' was characterized by fruit weight exceeding $5 \mathrm{~g}$. The mean mass of the fruits in the present experiments was higher than that found by Świerczyński and Stachowiak (2010). However, it must be stressed that with an increasing pruning intensity, the fruit weight increased. Pruning applications had no significant effect on firmness. Researchers obtained different results in sweet cherries (Bennewitz von et al., 2011). Fruit firmness was the highest after the application of moderate pruning. However, one should note that fruit firmness decreased with the progressing age of trees (Table 3 ). The only perceivable change under the increasing pruning intensity was the tendency to the decrease of TSS content in the fruit. Similar results were obtained by Zydlik et al. (2006), who reported that in the first years of tree growing, the tree crown shape did not exert any influence on fruit quality. 
Table 3. Effect of tree pruning intensity on the yield and fruit quality of sour cherry tree cultivar ' $\angle$ utówka' planted in 2002

\begin{tabular}{|c|c|c|c|c|c|c|}
\hline \multirow{3}{*}{ Treatment } & \multicolumn{5}{|c|}{ Years } & \multirow{2}{*}{$\begin{array}{c}\text { Mean for years } \\
2006-2010\end{array}$} \\
\hline & 2006 & 2007 & 2008 & 2009 & 2010 & \\
\hline & \multicolumn{6}{|c|}{ Yield kg tree ${ }^{-1}$} \\
\hline Without pruning & $8.52 \mathrm{ab}^{*}$ & $6.73 \mathrm{a}$ & $7.53 \mathrm{a}$ & $12.77 \mathrm{c}$ & $6.15 \mathrm{~b}$ & $8.34 \mathrm{~b}$ \\
\hline Moderate traditional pruning & $7.44 \mathrm{a}$ & $8.35 \mathrm{a}$ & $7.16 \mathrm{a}$ & $10.98 \mathrm{~b}$ & $4.47 \mathrm{ab}$ & $7.68 \mathrm{ab}$ \\
\hline Intensive pruning & $9.37 \mathrm{~b}$ & $6.74 \mathrm{a}$ & $6.42 \mathrm{a}$ & $9.47 \mathrm{a}$ & $3.40 \mathrm{a}$ & $7.08 \mathrm{a}$ \\
\hline \multirow[t]{2}{*}{ Mean for year } & $8.44 \mathrm{c}$ & $7.27 \mathrm{~b}$ & $7.04 \mathrm{~b}$ & $11.07 \mathrm{~d}$ & $4.67 \mathrm{a}$ & \\
\hline & \multicolumn{6}{|c|}{100 fruit weight $g$} \\
\hline Without pruning & $500.4 \mathrm{a}$ & $500.2 \mathrm{a}$ & $497.5 \mathrm{a}$ & $568.5 \mathrm{a}$ & $616.2 \mathrm{a}$ & $536.6 \mathrm{a}$ \\
\hline Moderate traditional pruning & $509.5 \mathrm{a}$ & $488.7 \mathrm{a}$ & $508.7 \mathrm{a}$ & $600.6 \mathrm{a}$ & $649.9 \mathrm{ab}$ & $551.5 \mathrm{ab}$ \\
\hline Intensive pruning & $485.0 \mathrm{a}$ & $517.0 \mathrm{a}$ & $550.7 \mathrm{~b}$ & $591.9 \mathrm{a}$ & $683.0 \mathrm{~b}$ & $565.5 \mathrm{~b}$ \\
\hline \multirow[t]{2}{*}{ Mean for year } & $498.3 \mathrm{a}$ & $502.0 \mathrm{a}$ & $519.0 \mathrm{a}$ & $587.0 \mathrm{~b}$ & $649.7 \mathrm{c}$ & \\
\hline & \multicolumn{6}{|c|}{ Firmness g } \\
\hline Without pruning & $267.2 \mathrm{a}$ & $214.7 \mathrm{a}$ & $189.0 \mathrm{a}$ & $163.5 \mathrm{a}$ & $196.0 \mathrm{~b}$ & $206.1 \mathrm{a}$ \\
\hline Moderate traditional pruning & $315.2 \mathrm{a}$ & $211.7 \mathrm{a}$ & $192.0 \mathrm{a}$ & $172.5 \mathrm{a}$ & $194.5 \mathrm{~b}$ & $217.2 \mathrm{a}$ \\
\hline Intensive pruning & $301.0 \mathrm{a}$ & $208.2 \mathrm{a}$ & $196.2 \mathrm{a}$ & $184.0 \mathrm{a}$ & $146.5 \mathrm{a}$ & $207.2 \mathrm{a}$ \\
\hline \multirow[t]{2}{*}{ Mean for year } & $294.5 \mathrm{c}$ & $211.5 \mathrm{~b}$ & $192.4 \mathrm{ab}$ & $173.3 \mathrm{a}$ & $179.0 \mathrm{a}$ & \\
\hline & \multicolumn{6}{|c|}{ Soluble solid content ${ }^{\circ}$ Brix } \\
\hline Without pruning & $15.77 \mathrm{~b}$ & $14.92 \mathrm{a}$ & $17.12 \mathrm{a}$ & $12.27 \mathrm{a}$ & $15.47 \mathrm{~b}$ & $15.11 \mathrm{a}$ \\
\hline Moderate traditional pruning & $15.05 \mathrm{a}$ & $14.90 \mathrm{a}$ & $17.42 \mathrm{a}$ & $12.27 \mathrm{a}$ & $14.57 \mathrm{a}$ & $14.84 \mathrm{a}$ \\
\hline Intensive pruning & $15.15 \mathrm{a}$ & $15.15 \mathrm{a}$ & $16.87 \mathrm{a}$ & $12.60 \mathrm{a}$ & $14.07 \mathrm{a}$ & $14.77 \mathrm{a}$ \\
\hline \multirow[t]{2}{*}{ Mean for year } & $15.32 \mathrm{c}$ & $14.99 \mathrm{bc}$ & $17.14 \mathrm{~d}$ & $12.38 \mathrm{a}$ & $14.70 \mathrm{~b}$ & \\
\hline & \multicolumn{6}{|c|}{ Acidity as the malic content $\%$} \\
\hline Without pruning & $1.73 \mathrm{a}$ & $1.53 \mathrm{a}$ & $1.73 \mathrm{a}$ & $1.85 \mathrm{a}$ & $1.81 \mathrm{a}$ & $1.73 \mathrm{a}$ \\
\hline Moderate traditional pruning & $1.68 \mathrm{a}$ & $1.51 \mathrm{a}$ & $1.69 \mathrm{a}$ & $1.77 \mathrm{a}$ & $1.75 \mathrm{a}$ & $1.68 \mathrm{a}$ \\
\hline Intensive pruning & $1.68 \mathrm{a}$ & $1.53 \mathrm{a}$ & $1.65 \mathrm{a}$ & $1.76 \mathrm{a}$ & $1.80 \mathrm{a}$ & $1.68 \mathrm{a}$ \\
\hline Mean for year & $1.70 \mathrm{~b}$ & $1.52 \mathrm{a}$ & $1.69 \mathrm{~b}$ & $1.78 \mathrm{~b}$ & $1.79 \mathrm{~b}$ & \\
\hline
\end{tabular}

Note. ${ }^{*}$ - different letters in the same column and for the same year represent significant differences at $5 \%$ level of significance.

Colour is a very important indicator of the quality of fresh fruit (Pedisić et al., 2009). During fruit ripening important biochemical changes modify the colour, texture, taste and other quality traits (Bureau et al., 2009). One of the aspects of the maturation of red fruits is the change of initial green colour to a red, violet or blackish colour, caused by the accumulation of anthocyanins and chlorophyll degradation (Pedisić et al., 2010). Colour variation of 'Łutówka' fruit skin was expressed by the $\mathrm{L}^{*}$, $\mathrm{a}^{*}, \mathrm{~b}^{*}, \mathrm{C}^{*}$ and $\mathrm{h}^{\circ}$ parameters (Tables 4-5). The changes in colorimetric parameters varied depending on year and orchard. The lower $\mathrm{C}^{*}$ value indicates an increase in

Table 4. Colour space of skin of cultivar ' $Ł u$ tówka', planted in 2001, depending on tree pruning intensity

\begin{tabular}{|c|c|c|c|c|c|c|}
\hline \multirow{2}{*}{ Year } & \multirow{2}{*}{ Treatment } & \multicolumn{5}{|c|}{ Colour components and indices } \\
\hline & & $\mathrm{L}^{*}$ & $a^{*}$ & $\mathrm{~b}^{*}$ & $\mathrm{C}^{*}$ & $\mathrm{~h}^{\circ}$ \\
\hline \multirow{3}{*}{2006} & Without pruning & $23.8 \mathrm{ab}$ & $20.0 \mathrm{a}$ & $2.8 \mathrm{a}$ & $20.2 \mathrm{a}$ & $6.3 \mathrm{a}$ \\
\hline & Moderate traditional pruning & $24.3 \mathrm{~b}$ & $21.9 \mathrm{a}$ & $3.5 \mathrm{a}$ & $22.2 \mathrm{a}$ & $7.3 \mathrm{a}$ \\
\hline & Intensive pruning & $22.9 \mathrm{a}$ & $22.3 \mathrm{a}$ & $3.4 \mathrm{a}$ & $22.5 \mathrm{a}$ & $7.0 \mathrm{a}$ \\
\hline \multirow{3}{*}{2007} & Without pruning & $19.6 \mathrm{a}$ & $12.1 \mathrm{a}$ & $0.2 \mathrm{a}$ & $12.2 \mathrm{a}$ & $1.0 \mathrm{a}$ \\
\hline & Moderate traditional pruning & $20.2 \mathrm{a}$ & $19.9 \mathrm{a}$ & $0.2 \mathrm{a}$ & $19.9 \mathrm{~b}$ & $0.5 \mathrm{a}$ \\
\hline & Intensive pruning & $19.8 \mathrm{a}$ & $19.2 \mathrm{a}$ & $-0.1 \mathrm{a}$ & $19.2 \mathrm{ab}$ & $-0.2 \mathrm{a}$ \\
\hline \multirow{3}{*}{2008} & Without pruning & $20.2 \mathrm{a}$ & $8.6 \mathrm{a}$ & $1.6 \mathrm{a}$ & $8.8 \mathrm{a}$ & $7.1 \mathrm{a}$ \\
\hline & Moderate traditional pruning & $20.1 \mathrm{a}$ & $9.4 \mathrm{a}$ & $1.7 \mathrm{a}$ & $8.6 \mathrm{a}$ & $7.1 \mathrm{a}$ \\
\hline & Intensive pruning & $20.4 \mathrm{a}$ & $8.3 \mathrm{a}$ & $1.7 \mathrm{a}$ & $8.5 \mathrm{a}$ & $7.6 \mathrm{a}$ \\
\hline \multirow{3}{*}{2009} & Without pruning & $20.5 \mathrm{~b}$ & $5.7 \mathrm{a}$ & $2.1 \mathrm{a}$ & $6.1 \mathrm{a}$ & $13.5 \mathrm{a}$ \\
\hline & Moderate traditional pruning & $20.3 \mathrm{ab}$ & $6.5 \mathrm{a}$ & $1.9 \mathrm{a}$ & $6.9 \mathrm{a}$ & $11.6 \mathrm{a}$ \\
\hline & Intensive pruning & $19.0 \mathrm{a}$ & $4.5 \mathrm{a}$ & $2.0 \mathrm{a}$ & $5.0 \mathrm{a}$ & $16.6 \mathrm{a}$ \\
\hline \multirow{3}{*}{2010} & Without pruning & $22.3 \mathrm{a}$ & $18.8 \mathrm{a}$ & $4.5 \mathrm{a}$ & $19.3 \mathrm{a}$ & $10.3 \mathrm{a}$ \\
\hline & Moderate traditional pruning & $22.3 \mathrm{a}$ & $15.8 \mathrm{a}$ & $4.5 \mathrm{a}$ & $16.5 \mathrm{a}$ & $11.6 \mathrm{a}$ \\
\hline & Intensive pruning & $22.8 \mathrm{a}$ & $16.2 \mathrm{a}$ & $5.1 \mathrm{a}$ & $17.0 \mathrm{a}$ & $12.9 \mathrm{a}$ \\
\hline \multirow{5}{*}{$\begin{array}{c}\text { Mean for } \\
\text { years }\end{array}$} & 2006 & $23.7 \mathrm{c}$ & $21.4 \mathrm{~d}$ & $3.2 \mathrm{c}$ & $21.6 \mathrm{~d}$ & $6.9 \mathrm{~b}$ \\
\hline & 2007 & $19.9 \mathrm{a}$ & $17.0 \mathrm{c}$ & $0.1 \mathrm{a}$ & $17.1 \mathrm{c}$ & $0.4 \mathrm{a}$ \\
\hline & 2008 & $20.2 \mathrm{a}$ & $8.8 \mathrm{~b}$ & $1.7 \mathrm{~b}$ & $8.9 \mathrm{~b}$ & $7.2 \mathrm{~b}$ \\
\hline & 2009 & $19.9 \mathrm{a}$ & $5.6 \mathrm{a}$ & $2.0 \mathrm{~b}$ & $6.0 \mathrm{a}$ & $13.9 \mathrm{~d}$ \\
\hline & 2010 & $22.5 \mathrm{~b}$ & $16.9 \mathrm{c}$ & $4.7 \mathrm{~d}$ & $17.6 \mathrm{c}$ & $11.6 \mathrm{c}$ \\
\hline \multirow{3}{*}{$\begin{array}{l}\text { Mean for } \\
\text { treatments }\end{array}$} & Without pruning & $21.3 \mathrm{a}$ & $13.0 \mathrm{a}$ & $2.2 \mathrm{a}$ & $13.3 \mathrm{a}$ & $7.7 \mathrm{a}$ \\
\hline & Moderate traditional pruning & $21.4 \mathrm{a}$ & $14.7 \mathrm{a}$ & $2.4 \mathrm{a}$ & $15.0 \mathrm{a}$ & $7.6 \mathrm{a}$ \\
\hline & Intensive pruning & $21.0 \mathrm{a}$ & $14.1 \mathrm{a}$ & $2.4 \mathrm{a}$ & $14.4 \mathrm{a}$ & $8.8 \mathrm{a}$ \\
\hline
\end{tabular}

Note. ${ }^{*}$ - different letters in the same column and for the same year represent significant differences at $5 \%$ level of significance. 
Table 5. Colour space of skin of cultivar 'Łutówka', planted in 2002, depending on tree pruning intensity

\begin{tabular}{|c|c|c|c|c|c|c|}
\hline \multirow{2}{*}{ Year } & \multirow{2}{*}{ Treatment } & \multicolumn{5}{|c|}{ Colour components and indices } \\
\hline & & $\mathrm{L}^{*}$ & $\mathrm{a}^{*}$ & $b^{*}$ & $\mathrm{C}^{*}$ & $\mathrm{~h}^{\circ}$ \\
\hline \multirow{3}{*}{2006} & Without pruning & $24.2 \mathrm{a}^{*}$ & $19.6 \mathrm{a}$ & $2.9 \mathrm{a}$ & $19.8 \mathrm{a}$ & $6.4 \mathrm{a}$ \\
\hline & Moderate traditional pruning & $23.9 \mathrm{a}$ & $22.7 \mathrm{~b}$ & $3.2 \mathrm{a}$ & $22.9 \mathrm{~b}$ & $6.7 \mathrm{a}$ \\
\hline & Intensive pruning & $24.4 \mathrm{a}$ & $22.5 \mathrm{~b}$ & $3.2 \mathrm{a}$ & $22.7 \mathrm{~b}$ & $6.6 \mathrm{a}$ \\
\hline \multirow{3}{*}{2007} & Without pruning & $18.8 \mathrm{a}$ & $15.2 \mathrm{~b}$ & $-0.2 \mathrm{a}$ & $15.2 \mathrm{~b}$ & $-0.6 \mathrm{a}$ \\
\hline & Moderate traditional pruning & $18.1 \mathrm{a}$ & $11.2 \mathrm{ab}$ & $1.7 \mathrm{ab}$ & $11.4 \mathrm{ab}$ & $6.4 \mathrm{a}$ \\
\hline & Intensive pruning & $17.8 \mathrm{a}$ & $7.9 \mathrm{a}$ & $3.2 \mathrm{~b}$ & $8.9 \mathrm{a}$ & $20.6 \mathrm{~b}$ \\
\hline \multirow{3}{*}{2008} & Without pruning & $21.1 \mathrm{a}$ & $7.6 \mathrm{a}$ & $1.5 \mathrm{a}$ & $7.8 \mathrm{a}$ & $7.4 \mathrm{a}$ \\
\hline & Moderate traditional pruning & $21.4 \mathrm{a}$ & $7.6 \mathrm{a}$ & $1.6 \mathrm{~b}$ & $7.8 \mathrm{a}$ & $7.9 \mathrm{a}$ \\
\hline & Intensive pruning & $21.5 \mathrm{a}$ & $7.4 \mathrm{a}$ & $1.6 \mathrm{~b}$ & $7.6 \mathrm{a}$ & $8.6 \mathrm{a}$ \\
\hline \multirow{3}{*}{2009} & Without pruning & $20.3 \mathrm{a}$ & $7.1 \mathrm{ab}$ & $2.9 \mathrm{a}$ & $7.7 \mathrm{ab}$ & $15.6 \mathrm{a}$ \\
\hline & Moderate traditional pruning & $20.3 \mathrm{a}$ & $9.0 \mathrm{~b}$ & $2.5 \mathrm{a}$ & $9.3 \mathrm{~b}$ & $11.0 \mathrm{a}$ \\
\hline & Intensive pruning & $20.1 \mathrm{a}$ & $5.3 \mathrm{a}$ & $2.3 \mathrm{a}$ & $5.8 \mathrm{a}$ & $16.2 \mathrm{a}$ \\
\hline \multirow{3}{*}{2010} & Without pruning & $22.4 \mathrm{a}$ & $13.7 \mathrm{a}$ & $4.2 \mathrm{a}$ & $14.3 \mathrm{a}$ & $12.3 \mathrm{a}$ \\
\hline & Moderate traditional pruning & $22.6 \mathrm{a}$ & $13.6 \mathrm{a}$ & $4.6 \mathrm{a}$ & $14.3 \mathrm{a}$ & $13.3 \mathrm{a}$ \\
\hline & Intensive pruning & $22.6 \mathrm{a}$ & $15.4 \mathrm{a}$ & $4.9 \mathrm{a}$ & $16.2 \mathrm{a}$ & $12.9 \mathrm{a}$ \\
\hline \multirow{5}{*}{$\begin{array}{l}\text { Mean for } \\
\text { years }\end{array}$} & 2006 & $24.2 \mathrm{e}$ & $21.6 \mathrm{~d}$ & $3.1 \mathrm{~b}$ & $21.8 \mathrm{~d}$ & $6.6 \mathrm{a}$ \\
\hline & 2007 & $18.2 \mathrm{a}$ & $11.4 \mathrm{~b}$ & $1.6 \mathrm{a}$ & $11.8 \mathrm{~b}$ & $8.8 \mathrm{ab}$ \\
\hline & 2008 & $21.3 \mathrm{c}$ & $7.5 \mathrm{a}$ & $1.6 \mathrm{a}$ & $7.7 \mathrm{a}$ & $7.9 \mathrm{ab}$ \\
\hline & 2009 & $20.2 \mathrm{~b}$ & $7.1 \mathrm{a}$ & $2.5 \mathrm{~b}$ & $7.6 \mathrm{a}$ & $14.3 \mathrm{~d}$ \\
\hline & 2010 & $22.5 \mathrm{~d}$ & $14.2 \mathrm{c}$ & $4.6 \mathrm{c}$ & $14.9 \mathrm{c}$ & $12.8 \mathrm{~cd}$ \\
\hline \multirow{3}{*}{$\begin{array}{l}\text { Mean for } \\
\text { treatments }\end{array}$} & Without pruning & $21.3 \mathrm{a}$ & $12.6 \mathrm{a}$ & $2.3 \mathrm{a}$ & $13.0 \mathrm{a}$ & $8.2 \mathrm{a}$ \\
\hline & Moderate traditional pruning & $21.2 \mathrm{a}$ & $12.8 \mathrm{a}$ & $2.7 \mathrm{a}$ & $13.2 \mathrm{a}$ & $9.0 \mathrm{a}$ \\
\hline & Intensive pruning & $21.3 \mathrm{a}$ & $11.7 \mathrm{a}$ & $3.0 \mathrm{a}$ & $12.2 \mathrm{a}$ & $13.0 \mathrm{~b}$ \\
\hline
\end{tabular}

Note. ${ }^{*}$ - different letters in the same column and for the same year represent significant differences at $5 \%$ level of significance.

tonality of the fruit colour (Gonçalves et al., 2007) and it was directly related to the pruning intensity in the orchard planted in 2001 (Table 3). The a* parameter depended on the year and orchard. The changes in fruit colour were best reflected by the $b^{*}$ coordinate representing the colours from dark green to magenta. The change in the b* coordinate was less distinct, but its growing tendency was observed for the increased intensity of pruning and for progressing tree age during 2007-2010. In addition, the Hue ${ }_{a b}$ value reflected the colour changes very clearly. These parameters decreased depending on tree pruning and its intensity. No consistent patterns in the chroma value were observed depending on pruning intensity.

\section{Conclusions}

1. The fruit yield decreased with the increasing tree pruning intensity. It must be stressed that fruit yield was the highest in the control treatment without pruning.

2 . The intensity of cherry tree pruning exerted an influence on the fruit weight, but it did not affect the fruit firmness, fruit extract content and acidity. Fruit firmness decreased with the progressing tree age.

3 . Both the measurements of the $b^{*}$ coordinate (representing the colours from blue to yellow) and the $\mathrm{Hue}_{\mathrm{ab}}$ angle increased with the intensity of the pruning but the differences were significant only for the trees planted in 2002 .

Received 11042015

Accepted 14092015

\section{References}

Bennewitz von E., Fredes C., Losak T., Martinez C., Hlusek J. 2011. Effects on fruit production and quality of different dormant pruning intensities in 'Bing'/'Gisela (R) 6' sweet cherries (Prunus avium) in Central Chile. Ciencia e Investigatión. Agraria, 38 (3): 339-344
Buler Z., Mika A. 2009. The influence of canopy architecture on light interception and distribution in 'Samion' apple trees. Journal of Fruit and Ornamental Plant Research, 17 (2): $45-52$

Bureau S., Renard C. M. G. C., Reich M., Ginies C., Andergon J. M. 2009. Change in anthocyanin concentrations in red apricot fruits during ripening. LWT-Food Science and Technology, 42: 372-377 http://dx.doi.org/10.1016/j.lwt.2008.03.010

Callesen O. 1997. Orchard systems for sour cherry. Acta Horticulturae, 451: 653-660 http://dx.doi.org/10.17660/ActaHortic.1997.451.77

Davarynejad G. H., Szabo Z., Persely S. Z., Szabo T., Nyéki J. 2014. The fruit set capability of some sour cherry cultivars (Prunus cerasus L.). Acta Horticulturae, 1020: 181-184 http://dx.doi.org/10.17660/ActaHortic.2014.1020.25

Flore J. A., Layne D. R. 1990. The influence of tree shape and spacing on light interception and yield in sour cherry (Prunus cerasus cv. Montmorency). Acta Horticulturae, 285: 91-96 http://dx.doi.org/10.17660/ActaHortic.1990.285.12

Gonçalves B., Silva A. P., Moutinho-Pereira J., Bacelar E., Rosa E., Meyer S. A. 2007. Effect of ripeness and postharvest storage on the evolution of colour and anthocyanins in cherries (Prunus avium L.). Food Chemistry, 103: 976-984 http://dx.doi.org/10.1016/j.foodchem.2006.08.039

Gonda I. 2006. The size of the canopy of sour cherry trees depends on the time of pruning. International Journal of Horticultural Science, 12 (3): 49-52

Gonda I., Such Z. 2008. Yielding capacity and regeneration of different ages fruit-bearing shoots in sour cherry. International Journal of Horticultural Science, 14 (1-2): $55-57$

Hrotkó K. 2009. Development of intensive orchard systems of stone fruits in Hungary. Acta Horticulturae, 825: 23-30 http://dx.doi.org/10.17660/ActaHortic.2009.825.1

Hrotkó K. 2013. Development in fruit trees production systems. AgroLife Scientific Journal, 2 (1): 28-35 
Hrotkó K., Csigai K., Magyar L., Hrotkó V. 2008 (a). Productivity of fruiting wood on slender spindle sour cherry trees. Acta Horticulturae, 795: 503-508 http://dx.doi.org/10.17660/ActaHortic.2008.795.77

Hrotkó K., Magyar L., Gyeviki M. 2008 (b). Achievements in high density cherry orchard systems in Hungary. Bulletin University of Agricultural Sciences and Veterinary Medicine Horticulture, 65 (1): 225-230

Ikinci A. 2014. Influence of pre- and postharvest summer pruning on the growth, yield, fruit quality, and carbohydrate content of early season peach cultivars. The Scientific World Journal, 8 p. http://dx.doi.org/10.1155/2014/104865

Jankiewicz L. S., Lipecki J. 2011. Physiology of orchard plants. Warszawa, Poland, p. 427-434 (in Polish)

Łysiak G. 2012. The base colour of fruit as an indicator of optimum harvest date for two apple cultivars (Malus domestica Borkh.). Folia Horticulturae, 24 (1): 81-89 http://dx.doi.org/10.2478/v10245-012-0012-2

Łysiak G., Kurlus R., Zydlik Z., Walkowiak-Tomczak D. 2014. Apple skin colour changes turing harvest as an indicator of maturity. Acta Scientiarum Polonorum, Hortorum Cultus, 13 (3): 71-83

Magyar L., Hrotkó K. 2013. The effect of rootstock and spacing on the growth and yield of 'Kántorjánosi 3' sour cherry variety in intensive orchard. Acta Horticulturae, 981: 373-378 http://dx.doi.org/10.17660/ActaHortic.2013.981.58

Mika A., Buler Z., Krawiec A. 2003. Effect various methods of pruning apple trees after planting. Journal of Fruit and Ornamental Plant Research, 11: 33-43

Mika A., Wawrzyńczak P., Buler Z., Krawiec A., Białkowski P., Michalska B., Plaskota M., Gotowicki B. 2011. Result of experiments with densely planted sour cherry trees for harvesting with a continuously moving combine harvester. Journal of Fruit and Ornamental Plant Research, 19 (2): 31-40

Pedisić S., Levaj B., Dragowić-Uzelac V., Škevin D., SkendrovićBabolejić M. 2009. Color parameters and total anthocyanins of sour cherries (Prunus cerasus L.) during ripening. Agriculturae Conspectus Scientificus, 74 (3): 259-262

Pedisić S., Levaj B., Dragowić-Uzelac V., Levaj B., Škevin D. 2010. Effect of maturity and geographical region on anthocyanin content of sour cherries (Prunus cerasus var. marasca). Food Technology Biotechnology, 48 (1): 86-93

Sredojević Z., Milić D., Jeločnik M. 2011. Investment in sweet and sour cherry production and new processing programs in terms of Serbian agriculture competitiveness. PetroleumGas University of Ploiesti Bulletin, 63 (3): 37-49

Stepulaitienè I., Žebrauskienė A., Stanys V. 2013. Frost resistance is associated with development of sour cherry (Prunus cerasus L.) generative buds. ZemdirbysteAgriculture, 100 (2): 175-178 http://dx.doi.org/10.13080/z-a.2013.100.022

Szpadzik E., Jadczuk-Tobiasz E., Łotocka B. 2008. Preliminary evaluation of pollen quality, fertility relations and fruit set of selected sour cherry cultivars in polish conditions. Acta Agrobotanica, 61 (1): 71-77 http://dx.doi.org/10.5586/aa.2008.009

Szpadzik E., Matulka M., Jadczuk-Tobiasz E. 2009. The growth, yielding and resistance to spring frost of nine sour cherry cultivars in central Poland. Journal of Fruit and Ornamental Plant Research, 17 (2): 139-148

Szpadzik E., Jadczuk-Tobiasz E., Łotocka B. 2010. Floral biology of some sour cherry cultivars and their suitability for cultivation. Horticulture and Landscape Architecture, 31: $43-51$

Świerczyński S., Stachowiak A. 2010. The influence of mycorrhizal fungi on the growth and yielding of plum and sour cherry trees. Journal of Fruit and Ornamental Plant Research, 18 (2): 71-77

Zydlik Z., Zachwieja M., Pacholak E., Rutkowski K. 2006. Effect of crown formation on the yielding and Ground Frost damages of sour cherry-trees. Publications of the Forestry and Agricultural Sciences Committee, 100: 317-324

ISSN 1392-3196 / e-ISSN 2335-8947

Zemdirbyste-Agriculture, vol. 102, No. 4 (2015), p. 417-422

DOI $10.13080 /$ z-a.2015.102.053

\title{
Genèjimo intensyvumo įtaka vyšnių vaisių derliui ir kokybei
}

\author{
K. Rutkowski, Z. Zydlik, E. Pacholak \\ Poznanès gyvybès mokslų universitetas, Lenkija
}

\section{Santrauka}

Tyrimo tikslas - ịvertinti medžių genėjimo intensyvumo ịtaką veislės 'Łutówka' vyšnių vaisių derliui ir kokybei. Eksperimentas buvo atliktas 2006-2010 m., taikant skirtingo intensyvumo vyšnių medžių genèjimą. Tirti vienų metų pirmos klasės skiepyti augalai: IR2 tipo veislès 'Łutówka' vyšnios su Prunus mahaleb poskiepiu, pasodintos

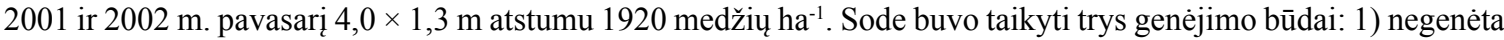
(pašalintos tik pažeistos šakos), 2) tradicinis vidutinis genėjimas (šakos tik patrumpintos), 3) intensyvus genejjimas (pašalintos senesnès nei 3 metų šakos). Vyšnių medžių derèjimas labai priklausė nuo eksperimento meteorologinių sąlygų. Genejjant intensyviau, vyšnių vaisių derlius mažèjo. Medžių genėjimas ir jo intensyvumas turèjo įtakos vaisių kokybei, kuri buvo vertinama pagal 100 vaisių svorị, vaisių tvirtumą, rūgštumą ir bendrą tirpių sausųų medžiagų kiekį. Veislès 'Lutówka' vyšnių vaisių odelès spalvos variacija buvo įvertinta pagal L*, $a^{*}, b^{*}, C^{*}$ ir $h^{\circ}$ spalvų koordinačių sistemą. Jų pokyčiai priklausė nuo metų ir sodo, kuriame augo vyšnios.

Reikšminiai žodžiai: derlius, genėjimas, Prunus cerasus, vainiko forma, vaisių kokybè, veislè. 